\title{
Deriving SOA Evaluation Metrics in an Enterprise Architecture Context
}

\author{
Stephan Aier ${ }^{1}$, Maximilian Ahrens ${ }^{2}$, Matthias Stutz ${ }^{1}$, and Udo Bub ${ }^{2}$ \\ ${ }^{1}$ University of St.Gallen, Switzerland \\ \{Stephan.Aier, Matthias.Stutz\}@unisg.ch \\ ${ }^{2}$ Deutsche Telekom Laboratories, Germany \\ \{Maximilian. Ahrens, Udo.Bub\} @ telekom. de
}

\begin{abstract}
Service oriented architectures (SOA) are becoming reality in a corporate environment. Rather than pure technology improvements SOA intends to increase manageability and sustainability of IT systems and to better align business requirements and technology implementations. Following these intentions current SOA research is focused on the management of SOA. In this paper we present a method to identify metrics for an evaluation of SOA. Therefore we adopted the goal/question/metrics method to SOA specifics. Since SOA is not limited to questions of technology we will show where SOA might be located in an enterprise architecture (EA) context. Based on the assumption that SOA follows different goals on different levels of EA abstraction, the paper shows, how these goals can be developed to metrics which can be consolidated in a measurement program.
\end{abstract}

\section{Introduction}

Service Oriented Architecture (SOA) is one of the recent approaches addressing the complexity of today's IT architectures. While SOA has been discussed in the scientific community, practitioners have also started to implement SOA in their organizations. However, organizations implementing SOA face different challenges, e.g. which are the goals to aim at, how to evaluate whether the implemented SOA achieved the defined goals, and - if not - which countermeasures should be taken? All of these questions are related to SOA management approach. Two essential elements of every management process are the measurement and evaluation of the current state.

In this paper we present a method to design a set of metrics to measure the success of SOA. With these metrics the architects have a set of indicators showing the impact of each of their decisions during the process of building and maintaining SOA. Thus architects will be better equipped to align the overall architecture with the set of desired qualities [1].

Our underlying research method is based on a design science approach [2]: After summarizing conceptual foundations we will review other approaches in the related work section and develop our method for the evaluation of SOA. We will also show 
how the method proposed can be applied to design a SOA measurement system. On the basis of several case studies and we will present our findings from applying the method in the final section.

\section{Conceptual Foundations}

The main idea of SOA is to encapsulate business functionality into small loosely coupled services which - once they are implemented on a software level - may be flexibly orchestrated to match the particular and ever changing business requirements. While SOA is often discussed from a technology perspective [3, 4], its actual potential is to align business requirements and processes with technical implementations [5]. This issue is regularly discussed as Business/IT alignment [6]. Therefore it is reasonable to link SOA to the concept of enterprise architecture (EA) in order to systematically address non-technical issues too [7]. Usually there is no single enterprise architecture but several partial architectures which may be organized in layers $[8,9]$. Based on an analysis of architecture meta-models Winter and Fischer [10] differentiate five layers with their respective architectures: Strategy layer (business architecture), organizational layer (process architecture), integration layer (integration architecture), and the system layers (software architecture, infrastructure architecture) (Fig. 1).

The integration layer is of particular importance. It couples artifacts of the software layer and the organizational layer. In the notion of SOA these couplings represent enterprise services [12]. These considerations outline the scope of the SOA management and measurement system. On the organizational layer the primary goals are effectiveness and efficiency [13], on the software layer the primary goal is reuse and

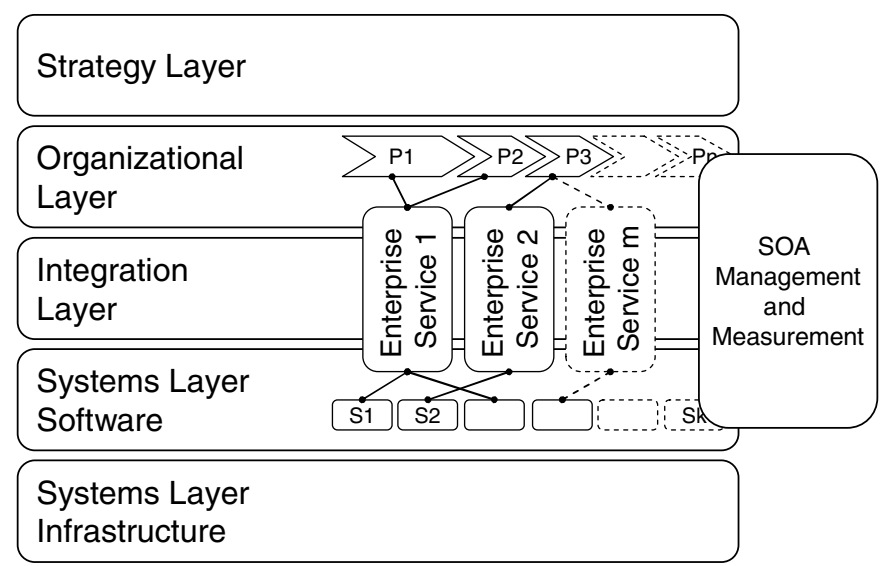

Fig. 1. Enterprise architecture, layers and services, cf. [11] 
on the integration layer the goals are alignment, adequate IT support for business processes, and ultimately agility [10, 14].

After we have analyzed the related work in the following section, we will present a method that will facilitate theses top level goals to a measurement program.

\section{Related Work}

Related work to the evaluation of SOA can be clustered in three different groups. The first group is formed by approaches that enable CIOs to argue in favor of the introduction of a SOA. These approaches are primarily sales-driven top down methodologies that allow rough estimations of financial benefits. These approaches are mostly presented by consulting companies [e.g. 15, 16] and often lack scientific methodology.

The second group of approaches primarily focuses on IT aspects of SOA evaluation. Many authors present approaches like the IT balanced scorecard to evaluate existing IT assets [17]. Other authors define and evaluate quality attributes which may refer to single applications [18] or to the entire IT architecture [19, 20].

The third group of related work can be identified in IT related extensions of process performance concepts, e.g. Gammelgård et al. propose to use the performance indicators of the CobIT framework [21]. Mooney et al. present a framework for process oriented business value measurement of IT combining existing approaches of the current literature [22].

Eventually, within the existing literature no methods for SOA evaluation in the context of EA exist. Either the approaches are driven from a technology perspective lacking a process and management view or they are driven from a business perspective not including technical aspects.

\section{Method Construction}

Our method to design a set of metrics to evaluate SOA focuses on the different goals SOA may pursue and takes the individual situation of the respective organization into account. Hence we propose to use a tailored version of the Goal/Question/ Metric (GQM) method [23].

GQM follows a top-down approach by first defining goals, then deriving questions and eventually deriving metrics. In our case of evaluating SOA the organizational layer, the integration layer and the software layer are affected. Although, as rooted in the nature of these layers, each layer has its separate goals, there is a hierarchy of goals according to the hierarchy of the layers. Strategic goals do affect the goals on the layers below. After the definition of goals, questions have to be derived which take the different aspects of a goal into account. Finally, based on these questions, metrics can be defined which are the basis for answering the questions. The questions in turn can be understood as guidelines of how to interpret the measured metrics (Fig. 2). 


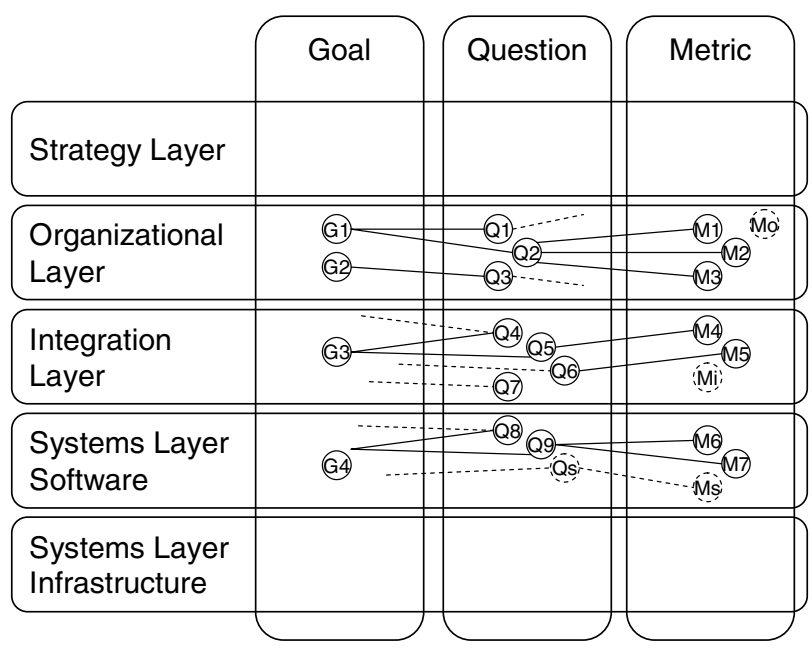

Fig. 2. Application of the Goal/Question/Metric method in the context of SOA

Following the GQM method a set of metrics has to be tailored to fit the individual situation. In order to raise the quality and acceptance of the measurements this will be done in structured interviews with the respective stakeholders. In accordance with van Latum et al. we introduce so called abstraction sheets to facilitate these interviews (Fig. 3). These abstractions sheets are the basis for deriving questions and metrics [24].

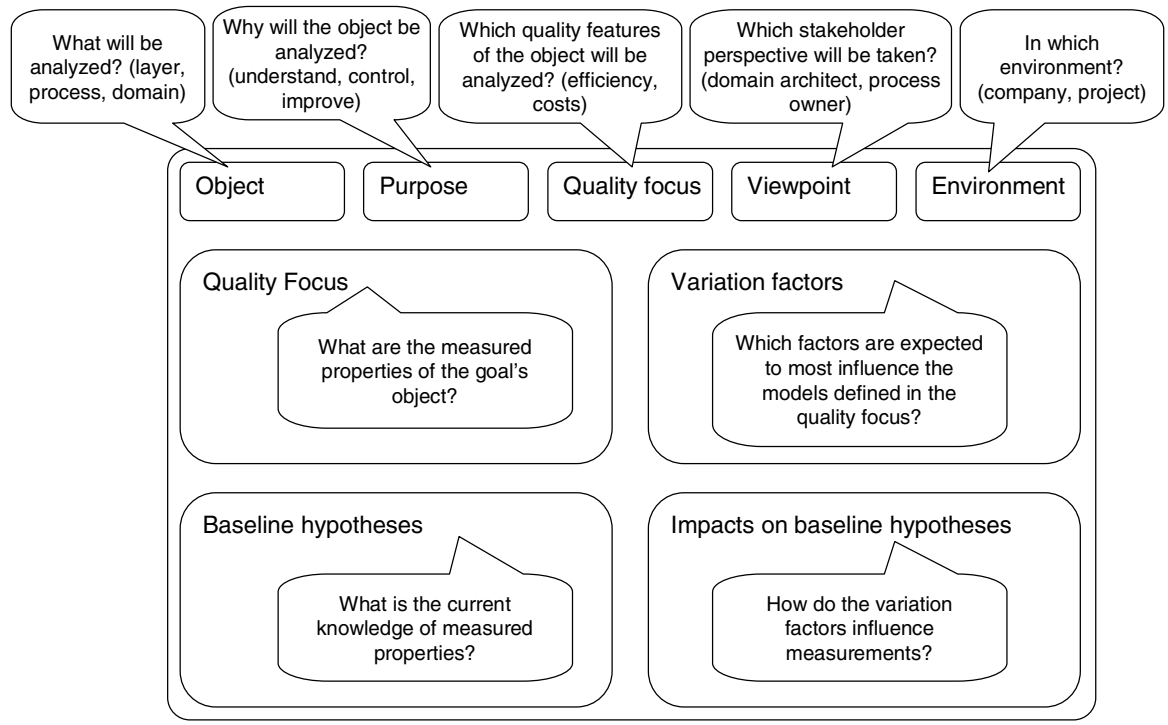

Fig. 3. Example GQM abstraction sheet, cf. [24] 
After defining goals to questions to metrics these metrics have to be consolidated in a measurement program. The aim of the consolidation is to reduce the number of metrics by isolating independent metrics and focusing on metrics which are applicable for answering several questions. In the EA environment this step requires a more formal approach than the one being used in the standard GQM method. This is caused by the highly complex environment as well as the involvement of many different stakeholders. We propose to use Extended Influence Diagrams (EID) for this formalization step (Fig. 7). The goal of the GQM method is depicted as a utility node within the influence diagram. Nodes within an EID can be connected by two different relationship types: causal and definitional.

Within the EID uncertainty of the chance nodes can be addressed by adding probability information to the nodes. This allows to reflect the quality of the metrics and to differentiate between directly and indirectly measurable metrics. For example if the goal is to have a very much quantifiable analysis, the influence of the indirectly measurable metrics could be reduced. It is important to analyze, whether all of the consolidated metrics are actually measurable, i.e. the metrics have to be available with a reasonable effort.

Eventually a measurement program has to define, how the set of metrics will be embodied in the organization, i.e. who will when measure which metrics.

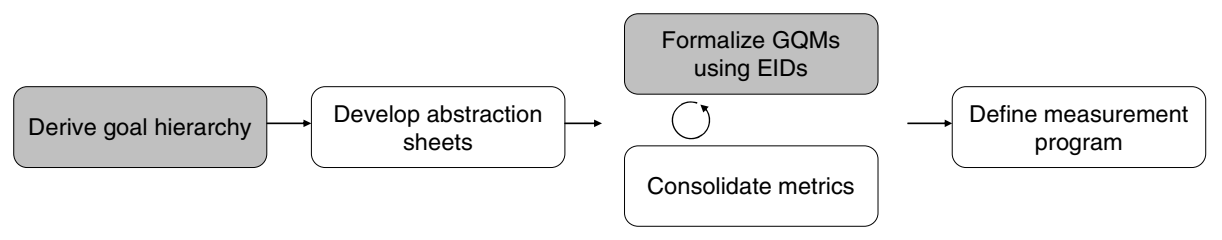

Fig. 4. Adopted GQM method, additional steps shaded grey

\section{Application of the Method}

This section describes the evaluation results and exemplarily shows a number of resulting artifacts of the method application. We primarily refer to a case study that has been conducted in Deutsche Telekom, which is the largest German telecommunications provider. The method was used within a project that aimed at understanding the current state of enterprise services and on identifying potentials for improvement. The actual project used a traditional portfolio approach without employing the adopted GQM method. To evaluate our method we ex post used the project data with the original project results as a benchmark to our method. According to the presented method in the first step a goal hierarchy has been developed. A simplified example is shown in Fig. 5.

The implementation of the adapted GQM method has been supported by the abstraction sheet, shown in Fig. 6.

As the first step within the evaluation method, object and purpose need to be defined. We defined the Enterprise Service 1 including related software objects $\left(\mathrm{S}_{\mathrm{i}}\right)$ and process segments $\left(\mathrm{P}_{\mathrm{j}}\right)$ of the layered business model as depicted in Fig. 1. 


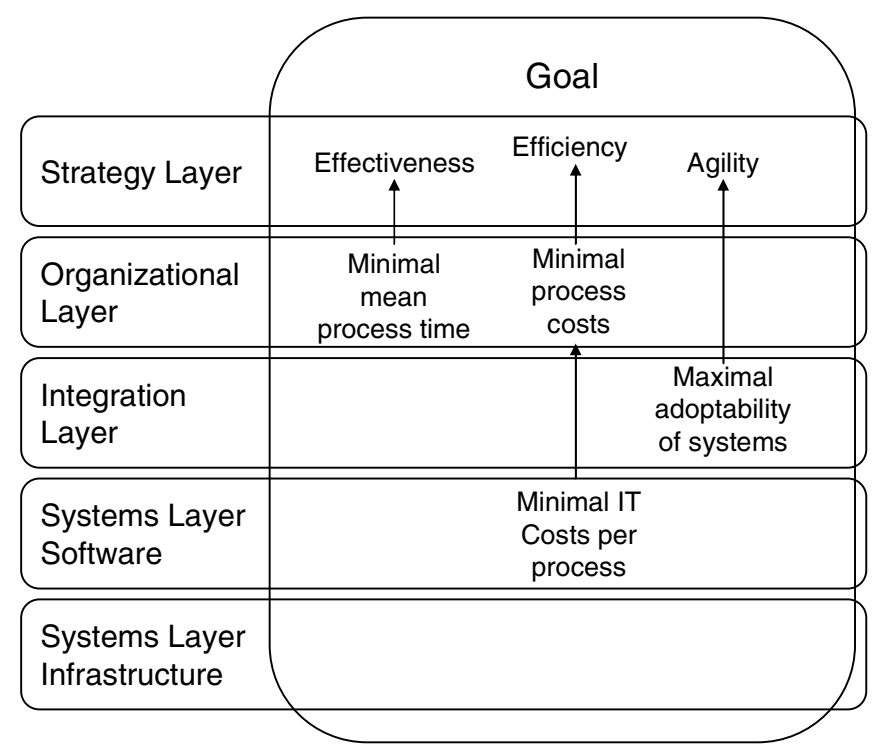

Fig. 5. Case study goal hierarchy

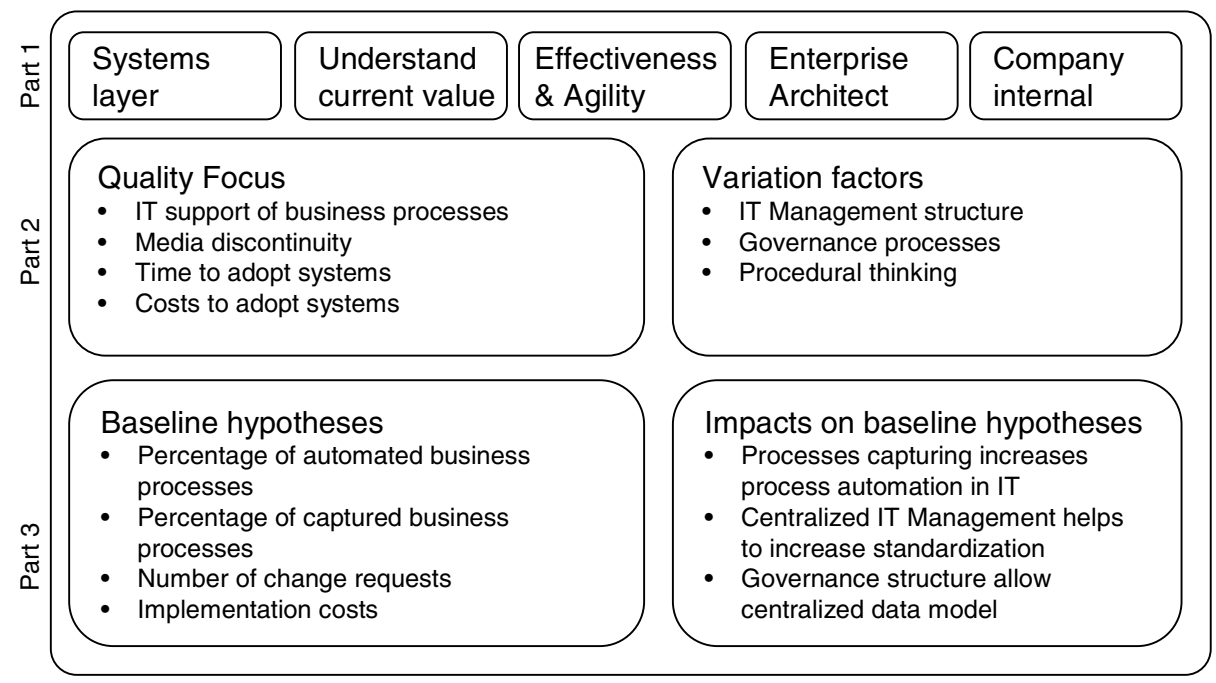

Fig. 6. Simplified abstraction sheet

Part two of the abstraction sheet defines the questionnaire outline. The detailed quality focus tries to find the characteristics of the top level quality. In our analysis the effectiveness goal has been divided into several sub-characteristics, like media discontinuity, adoption costs, etc. These attributes define the quality focus more precisely and later on help to define the actual metrics. 
Part three of the abstraction sheet describes the as-is analysis of the artifact. The abstraction sheet guides the interviewee to metrics and the assumed impact of the variation factors on the measures of the baseline hypothesis.

First the questions are clustered and condensed to the most relevant question within each of the clusters. The second step is the assignment of the metrics that have been identified. To do so the baseline hypothesis and the impacts on it are analyzed. An example for an indirectly measurable metric is shown in the question depicted in Fig. 8.

For the formal representations of the impact factors we use EIDs (Fig. 7).

After having defined the GQM hierarchy the measurement plan is defined, containing measurement policies and organizational structures. For every metric the measurement frequency, the roles responsible for measuring and the actual measurement process have to be defined.

As a result of the case study we found that the structured approach of the GQM method allows building a consistent and focused set of metrics that are linked with

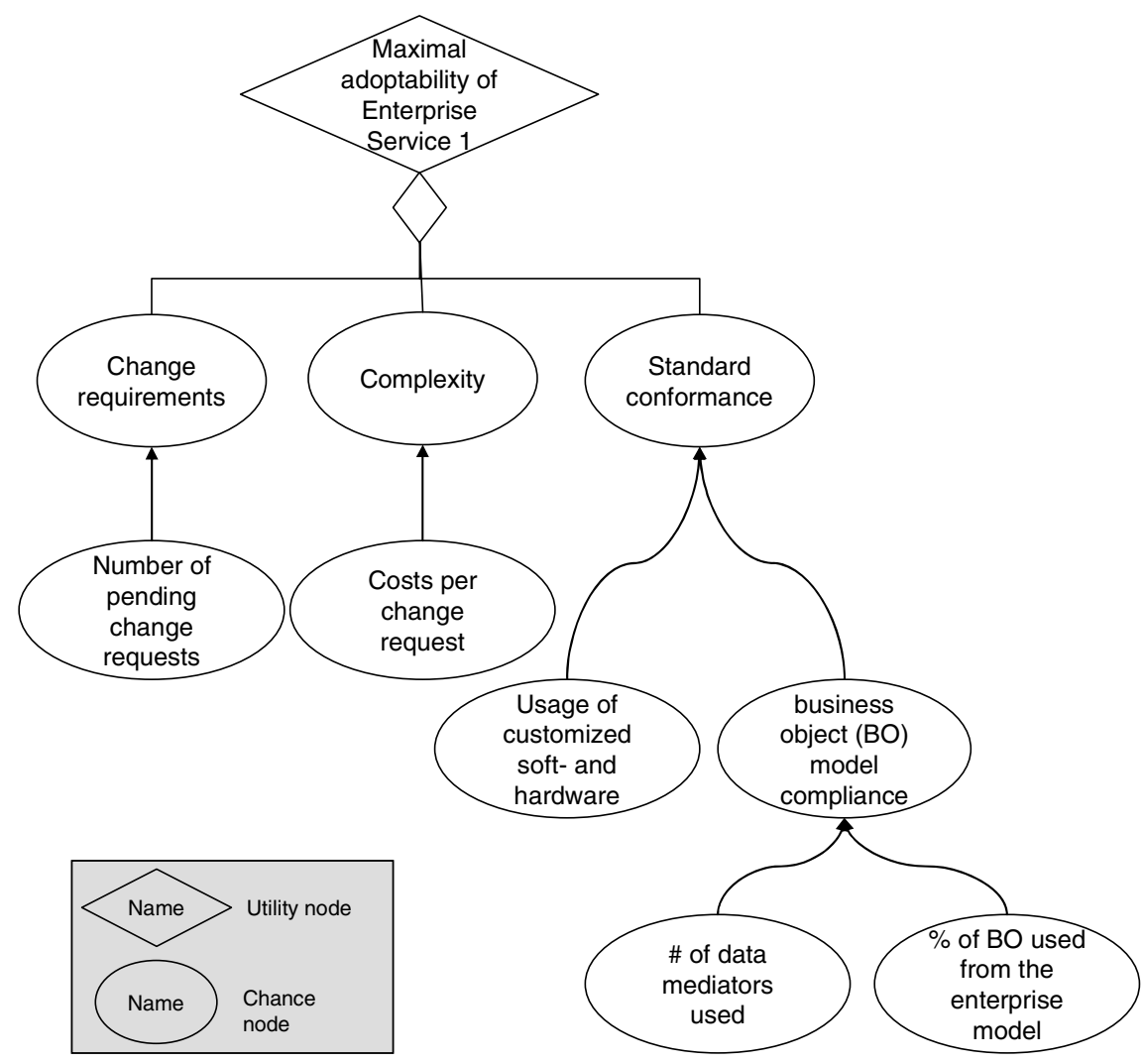

Fig. 7. GQM EID hierarchy 


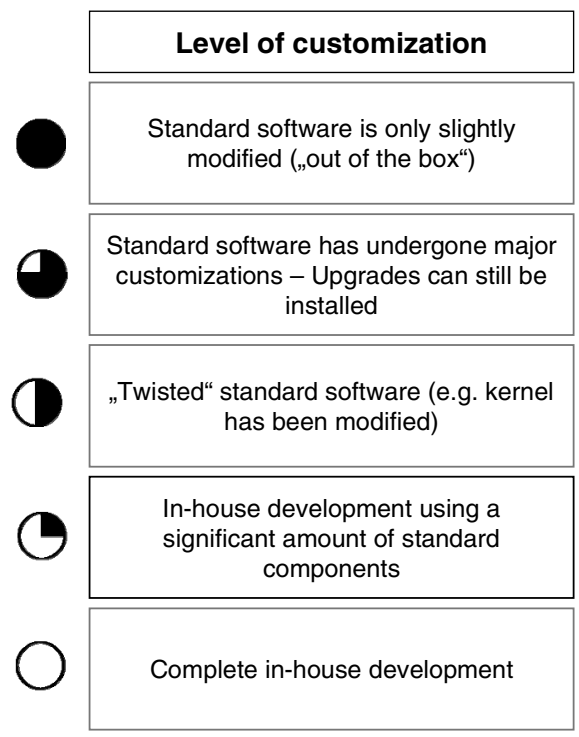

Fig. 8. Example questionnaire for measuring the customization level

goals which are derived from strategic viewpoints. When comparing the method presented here to other approaches, several advantages were found, three of which should be briefly named. The first improvement is the usage of goal hierarchies which allow consistency management of the various goals within the EA. Secondly the formalization approach allows a more precise consolidation and comparison of the used metrics. Third, after discussing the approach with the stakeholders involved in the original project we found, that this structured, formalized, and thus transparent approach created a much higher acceptance of the metrics among the stakeholders.

\section{Summary and Outlook}

In this paper we have described a method for the definition of metrics that can be utilized to evaluate SOA. Our method is based on the GQM method and thus ensures to deliver situational metrics which are derived systematically. However, we assume that it is possible to identify certain SOA project types and certain context factors which can be combined to situations. If it is possible to identify an appropriate number of relevant situations, we could define a number of reference metrics which were immediately available for application. So our next research activities will focus on the identification of relevant project types and context factors.

\section{References}

1. Vasconcelos, A., Sousa, P., Tribolet, J.: Information System Architecture Metrics: An Enterprise Engineering Evaluation Approach. In: The 13th European Conference on Information Technology Evaluation (ECITE 2006) (2006) 
2. Hevner, A.R., et al.: Design Science in Information Systems Research. MIS Quarterly 28(1), 75-105 (2004)

3. Gold, N., et al.: Understanding Service-Oriented Software. IEEE Software 21(2), 71-77 (2004)

4. W3C. Web Services Glossary. W3C Working Group Note (2004) [cited 02.01.2005], http: / /www.w3 . org/TR/2004/NOTE-ws-gloss-20040211

5. Rabhi, F.A., et al.: A service-oriented architecture for financial business processes. Information Systems And E-Business Management 5(2), 185-200 (2007)

6. Luftman, J.N., McLean, E.R.: Key Issues for IT Executives. MIS Quarterly Executive 3(2), 89-104 (2004)

7. Lankhorst, M.: Enterprise Architecture at Work: Modelling, Communication and Analysis. Springer, Berlin (2005)

8. The Open Group, TOGAF (The Open Group Architecture Framework) Version 8.1 Enterprise edn., San Francisco, CA (2003)

9. CIO-Council. Federal Enterprise Architecture Framework Version 1.1 (1999) [cited 31.10.2006], http: / / www. cio.gov/archive/fedarch1.pdf

10. Winter, R., Fischer, R.: Essential Layers, Artifacts, and Dependencies of Enterprise Architecture. Journal of Enterprise Architecture 3(2), 7-18 (2007)

11. Winter, R., Fischer, R.: Essential Layers, Artifacts, and Dependencies of Enterprise Architecture. In: EDOC Workshop on Trends in Enterprise Architecture Research (TEAR 2006). IEEE Computer Society, Los Alamitos (2006)

12. Schelp, J., Winter, R.: Towards a Methodology for Service Construction. In: Proceedings of the 40th Hawaii International Conference on Systems Sciences (HICSS-40). IEEE Computer Society, Los Alamitos (2007)

13. Österle, H.: Business in the Information Age - Heading for New Processes. Springer, New York (1995)

14. Yusuf, Y.Y., Sarhadi, M., Gunasekaran, A.: Agile manufacturing: the drivers, concepts and attributes. International Journal of Production Economics 62(1-2), 33-43 (1999)

15. DiMare, J.: Serviceoriented architecture: A practical guide to measuring return on investment (2006) [cited 24.05.2007],

http: //www-935.ibm.com/services/de/bcs/pdf/2007/

soa_practical_guide.pdf

16. Barnes, M.: Applied SOA: Measuring Business Value (2006) [cited 24.05.2007], http://www.gartner.com/teleconferences/attributes/attr_150040_115.pdf

17. Son, S., Weitzel, T., Laurent, F.: Designing a Process-Oriented Framework for IT Performance Management Systems. The Electronic Journal Information Systems Evaluation 8(3), 219-228 (2005)

18. Johnson, P., et al.: Extended Influence Diagrams for Enterprise Architectural Analysis. In: Proceedings of the 10th IEEE International Annual Enterprise Distributed Object Computing Conference (2006)

19. Vasconcelos, A., et al.: An information system architectural framework for enterprise application integration. In: Proceedings of the 37th Annual Hawaii International Conference on System Sciences (HICSS 2004). IEEE Computer Society, Los Alamitos (2004)

20. Khaddaj, S., Horgan, G.: The Evaluation of Software Quality Factors in Very Large Information Systems. Electronic Journal of Information Systems Evaluation 7(1), 43-48 (2004)

21. Gammelgård, M., et al.: Business Value Evaluation of IT Systems: Developing a Functional Reference Model. In: Proceedings of the Conference on Systems Engineering Research (CSER 2006). INCOSE, Los Angeles (2006) 
22. Mooney, J.G., Gurbaxani, V., Kraemer, K.L.: A process oriented framework for assessing the business value of information technology. ACM SIGMIS Database 27(2), 68-81 (1996)

23. Basili, V.R., Weiss, D.M.: A Methodology for Collecting Valid Software Engineering Data. IEEE Transactions on Software Engineering 10(6) (1984)

24. Van Latum, F., et al.: Adopting GQM based measurement in an industrial environment. IEEE Software 15(1), 78-86 (1998) 